\title{
Complex Odontoma - A Case Report
}

\author{
B. Pavan Kumar, Koduru Nikhila, G. Srinivas, V. Vidya Devi \\ Department of Oral and Maxillofacial Surgery, Kamineni Institute of Dental Sciences, Nalgonda, Telangana, India
}

Email for correspondence: koduru.nikki@gmail.com

\begin{abstract}
Odontoma refers to tumor of odontogenic origin. It represents a hamartomatous malformation rather than a neoplasm. Calcified dental tissues are simply an irregular mass bearing no morphologic similarity even to rudimentary teeth known as complex odontoma. Complex odontoma appears radiographically as a radiopaque mass. Complex form of odontoma is less common than compound type. Here, we report such an unusual case of complex odontoma.
\end{abstract}

Key words: Complex odontoma, odontogenic tumor, odontoma

\section{INTRODUCTION}

Teeth develop from a complex interaction between ectodermal and ectomesenchymal tissues. Remnants of these tissues are left behind in the jaws both during and after odontogenesis and can give rise to a heterogeneous group of odontogenic lesions. ${ }^{[1]}$ According to the World Health Organization (WHO), histologic classification of odontogenic tumors, these tumors fall into three categories: $:^{[2]}$

- Tumors originating from odontogenic epithelium without odontogenic ectomesenchyme

- Tumors originating from odontogenic epithelium with odontogenic ectomesenchyme, with or without dental hard tissue

- Tumors originating from odontogenic ectomesenchyme with or without odontogenic epithelium.

Odontomas are considered to be developmental anomalies resulting from the growth of differentiated epithelial and mesenchymal cells. ${ }^{[3]}$ The term odontoma (or odontome) first described by Paul Broca, in 1867. On the basis of gross, radiographic, and microscopic features, two types of odontoma are recognized: The compound and the complex. ${ }^{[4]}$ These

\begin{tabular}{|l|l|}
\hline Quick Response Code & Article Info: \\
\hline doi: 10.5866/2019.11.10112 & $\begin{array}{l}\text { Received: } 16-07-2019 \\
\text { Revised: } 17-08-2019 \\
\text { Accepted: } 24-08-2019 \\
\text { Available Online: 01-10-2019, (www. } \\
\text { nacd.in) ( ) NAD, } 2019 \text { - All rights reserved }\end{array}$ \\
\hline
\end{tabular}

are composed of more than one type of tissue and hence termed as composite odontoma. ${ }^{[5]}$ Complex odontomas are less common than the compound variety in the ratio of 1:2. ${ }^{[6]}$ The WHO, in 2005, categorized odontomas as calcified irregular mass not resembling teeth, as complex odontome and calcified mass bearing resemblance to the teeth, as compound odontome. ${ }^{[7]}$

The present case report presents an unusual case of complex odontoma in a 40-year-old female, presenting as a radiopaque and radiolucent mass at the apex of the left lower second and third molar tooth on radiograph. Written informed consent was obtained from the patient for publication of this report and any accompanying images.

\section{CASE REPORT}

A 40-year-old woman visited the department of oral and maxillofacial surgery with swelling in the left buccal vestibule that had persisted for approximately 1 month. The patient's medical history was unremarkable, and there was no reported history of maxillofacial trauma.

The swelling was reported to have increased over 2 weeks and causing discomfort while eating. No signs of intraoral and extraoral discharge present.

The clinical examination revealed a gross asymmetry due to swelling in the left lower onethird region of the face [Figures 1 and 2]. A solitary ill-defined swelling was present in the left lower third of the face, measuring approximately $4 \mathrm{~cm} \times 5 \mathrm{~cm}$ in size. It extended mesiodistally from the left parasymphysis region to angle of the 
mandible and superio-inferiorly $1.5 \mathrm{~cm}$ below from ala-tragus line to $2.0 \mathrm{~cm}$ and below the inferior border of the mandible. Overlying skin was normal with no history of any kind of discharge or pulsation. Palpatory findings confirmed that the swelling was tender, bony hard, well-defined margins, smooth surface, and with no local temperature rise. Palpation of the cervical lymph nodes and neck did not reveal any abnormality.

Intraorally, a solitary swelling was present in the left mandibular region irt 36 and 37, involving the buccal vestibule, with a size of approximately $2 \mathrm{~cm} \times 2 \mathrm{~cm}$, extending mesiodistally from distal aspect of 35-1.5 cm anterior to the retromolar region. Swelling was mild tender, bony hard in consistent, non-mobile, non-fluctuant, and compressible

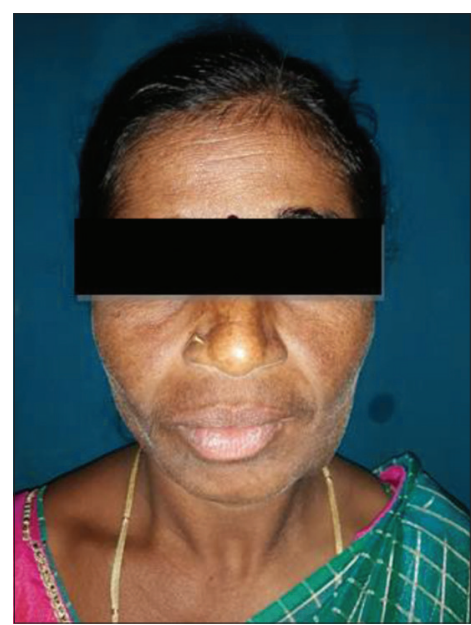

Figure 1: Frontal view of swelling in the left lower one-third region of the face

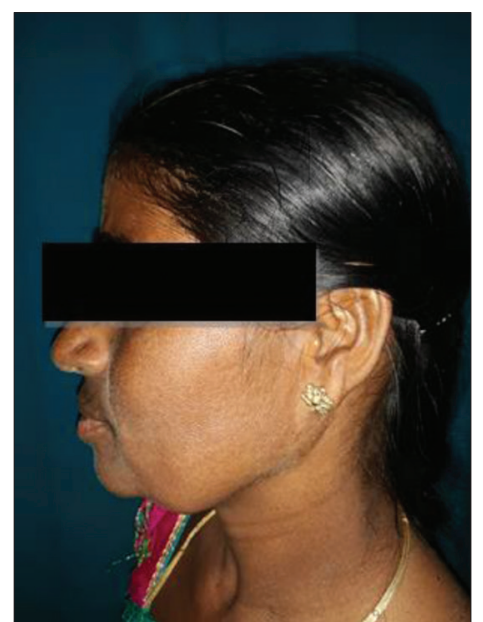

Figure 2: Lateral view of swelling in the left lower one-third region of the face with diffused borders [Figure 3]. On radiographic examination, orthopantomogram showed a lesion present irt 36 and 37 with irregular radiolucent borders. The lesion was largely radiopaque with irregular mass of calcified tissue, the radiopacity of which exceeded that of adjacent tooth structures [Figure 4].

Clinical and radiological examination led to the diagnosis of complex odontoma and surgical excision of the mass with the involved tooth was planned. Under general anesthesia, the lesion was removed surgically with extraction of the involved 36, 37, and 38 through an intraoral approach and packed with betadine pack dressing to avoid dead space. The surgical specimen was sent for histopathological examination [Figure 5]. Based on the histopathological examination, an irregular arrangement of dental tissues such as enamel, dentin, cementum, and together with odontoblastic cells, the diagnosis of complex odontoma was confirmed [Figure 6].

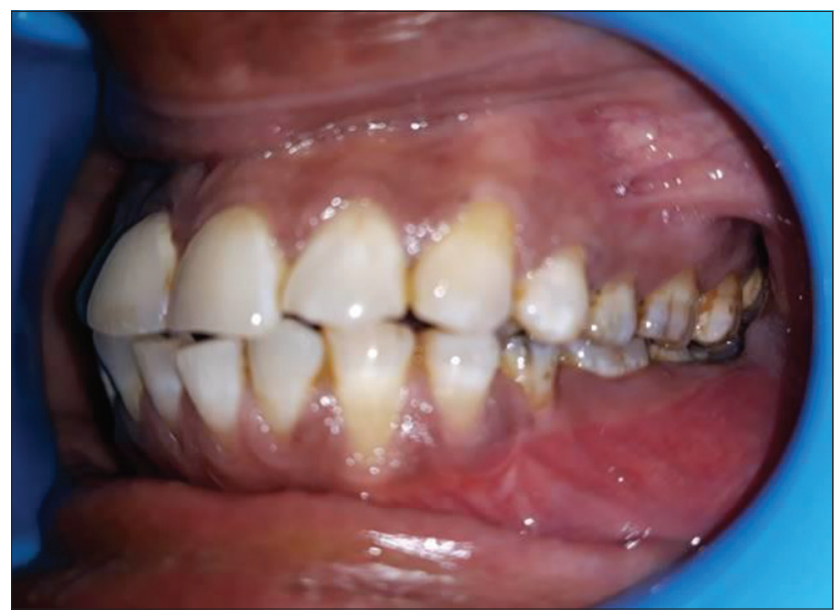

Figure 3: Intraorally, a solitary swelling in the left mandibular region irt 36 and 37

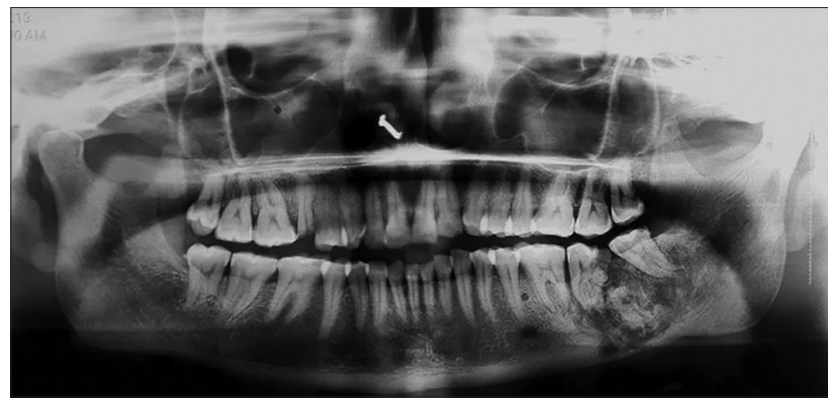

Figure 4: Orthopantomogram showing a lesion present irt 36 and 37 


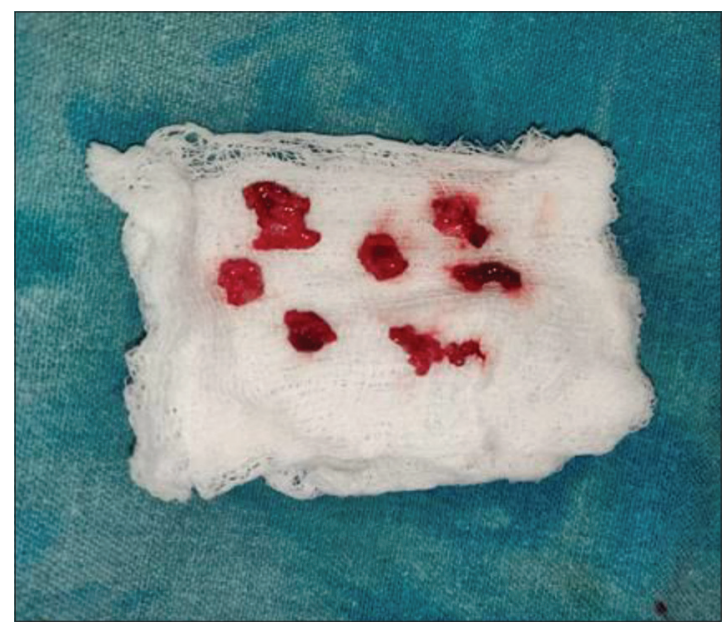

Figure 5: Surgical specimen sent for histopathological examination

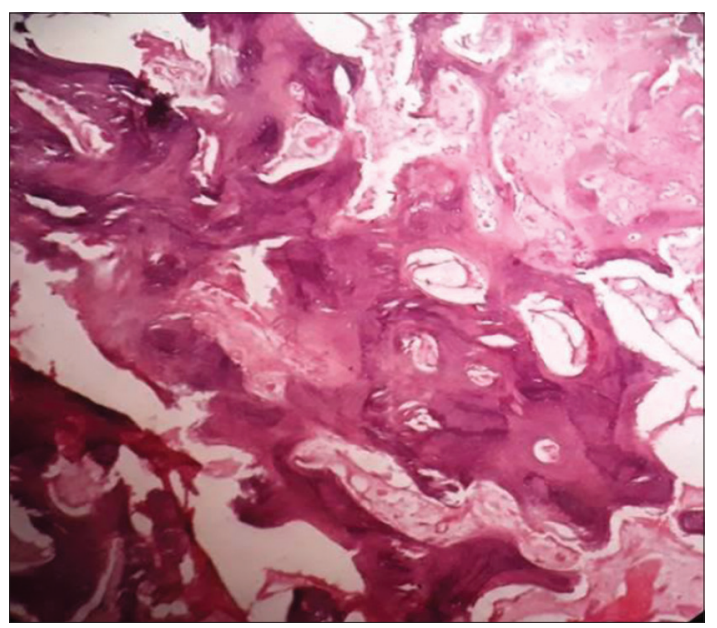

Figure 6: Photomicrograph of $\mathrm{H}$ and $\mathrm{E}$ stained section shows calcified dentin, enamel, and cementum in irregularly arranged pattern suggestive of complex odontoma

\section{DISCUSSION}

Broca first coined the term "odontome," in 1866. He defined it as a tumor formed by an overgrowth of complete dental tissue. ${ }^{[8]}$ This term was expanded overtime to include many odontogenic tumors or cysts. In a landmark paper, Thoma and Goldman narrowed the term "odontoma" to include tumors that were composed of well-differentiated tooth structure. ${ }^{[9]}$ Shafer and Gorlin defined odontoma as a tumor that has developed and differentiated enough to produce enamel and dentin. ${ }^{[10,11]}$ Even in the most differentiated form of odontoma, the compound odontoma, the toothlike material is laid down in an abnormal pattern due to the failure of the progenitor odontogenic cells to reach a full state of morphodifferentiation. ${ }^{[10]}$

\section{Etiology}

Some odontomas are associated with a history of trauma during primary dentition, as well as with inflammatory and infectious processes, hereditary anomalies (Gardner's syndrome and Hermann's syndrome), odontoblastic hyperactivity, or alterations of the genetic components responsible for controlling tooth development. ${ }^{[12]}$

One aspect of the etiology of odontomas is odontogenic epithelial cells. ${ }^{[13]}$ When these buds are divided into several particles, they may develop individually to become numerous, closely positioned malformed teeth or tooth-like structures. When the buds develop without such uncommon divisions and consist of haphazard of dental tissues, they may develop into complex odontomas. The transition from one type to another is commonly associated with varying degrees of morphodifferentiation or histodifferentiation. ${ }^{[14]}$ Persistence of a portion of lamina may be an important factor in the etiology of a compound odontoma.

\section{Incidence}

The complex odontomas are slow growing, expanding lesions that are usually detected in the second decade of life. The lesions are invariably asymptomatic and are usually discovered on routine radiographic examinations. ${ }^{[4]}$ These odontogenic tumors can be found anywhere in the dental arches. The majority of odontomas which are located in the anterior region of the maxilla are compound, while the great majority of odontomas located in the posterior areas, especially in the mandible, are complex odontomas. ${ }^{[3]}$ Pain, swelling, and infection were the most common symptoms. ${ }^{[15]}$ Odontomas have no sex predilection and are mostly diagnosed in the second decade of life. ${ }^{[16]}$

\section{Diagnosis}

In the case of typical compound odontomas, there are comparatively large, well-organized malformed teeth or tooth-like structural, complex odontomas consist of less well-organized dental tissues that seldom show teeth or tooth-like structures. ${ }^{[17]}$ A visual examination of the lesions cannot by itself define the differences between the complex and compound types because the odontomas are usually lie in the bone and do not show outward signs, such as expansion of the bone. Even in the rare instances, in which odontomas erupt into the oral cavity and can be examined visually and manually, the surface appearances of both types of 
odontoma are similar and differentiation between them is difficult. ${ }^{[18]}$ The complex odontoma presents a disorderly arrangement of all tissues although complete histodifferentiation has occurred. A connective tissue capsule around the odontoma is always present.

In comparison with visual examination and manual palpation, radiographic examination seems to be the most effective clinical method of discriminating between the two types. ${ }^{[19]}$ In case of compound odontome, well-organized malformed teeth or tooth-like structure is seen. Complex odontoma consists of less well-organized dental tissue. A visual examination of the lesions cannot by itself define the differences between complex and compound odontoma because the surface appearances that most result from extraneous buds of both types of odontoma are similar and differentiation between them is difficult.

To establish a definite diagnosis, some other procedure must be used, most commonly. Microradiography is another useful procedure in the effort to establish a definite diagnosis. It enables histologic structures to be recognized from their various radiopacities and/or radiolucencies. In addition, the resolution of microradiography is equal to that obtained in histologic examinations. ${ }^{[18]}$

Radiographically, odontoma presents as a well-defined radiopacity situated in bone but with a density that is greater than bone and equal to or greater than that of a tooth. It contains foci of variable density. It is present with a radiolucent halo, typically surrounded by a thin sclerotic line, surrounding the radiopacity. Radiolucent zone is the connective tissue capsule of a normal tooth follicle. Thin sclerotic line resembles the corticated border seen in a normal tooth crypt. Developmental stages can be identified based on radiologic features and the degree of calcification of the lesion at the time of diagnosis. First stage is characterized by radiolucency due to the absence of dental tissue calcification, the second or intermediate stage shows partial calcification, and the third or classically radiopaque stage exhibits predominant tissue calcification with the surrounding radiolucent halo. ${ }^{[20]}$ The complex odontoma presents as a well-demarcated radiopaque mass, occasionally surrounded by a narrow, radiolucent zone. ${ }^{[21]}$ In compound odontoma, multiple dense radiopaque structure, with clear external margins presenting normal organization of dental tissues, i.e., enamel, dentin, and pulp. ${ }^{[20]}$
Histologically, odontomas are composed of various dental tissue formations, including enamel, dentin, cementum, and sometimes also pulp. Complex odontomas show primary or immature dentin as the predominant component, though enamel is also found, exhibiting two possible types of distribution: A highly calcified area close to the central core or a hypocalcified zone with immature enamel. Immature cement is also observed together with the external connective tissue capsule surrounding the lesion, resembling the dental follicular tissue. Complex odontomas have been associated with unerupted teeth. ${ }^{[21]}$ In compound odontoma decalcified dentin, decalcified enamel intermixed with haphazardly arranged areas containing remnants of immature enamel matrix. ${ }^{[20}$

\section{Treatment}

The treatment indicated for the odontoma is surgical excision. The fibrous capsule facilitates dissection of the lesion away from the surrounding bone and the frequency of recurrence is minimal; the prognosis is excellent. ${ }^{[22]}$

\section{CONCLUSION}

A case of complex odontoma has been reported. The most interesting feature, in this case, was that the complex odontoma occurred at the fourth decade of life which is rare in occurrence and associated with pain. A surgical intervention was done and the odontomas were extracted.

\section{REFERENCES}

1. Melrose RJ. Benign epithelial odontogenic tumors. Semin Diagn Pathol 1999;16:271-87.

2. Kramer IR, Pindborg JJ, Shear M. Histological Typing of Odontogenic Tumours. $2^{\text {nd }}$ ed. Berlin: Springer-Verlag; 1992.

3. Siddhartha SV, Freny K. Complex odontome: A review and case report. Int J Adv Health Sci 2014;1:21-5.

4. Tiwari A, Srinivas K. Complex odontome-a rare case report. Am J Oral Med Rad 2016;3:119-21.

5. Prabhakar C, Haldavnekar S, Hegde S. Compound-complex odontoma-an important clinical entity. J Int Oral Health 2012;4:49-53.

6. Vengal M, Arora H, Ghosh S, Pai KM. Large erupting complex odontoma: A case report. J Can Dent Assoc 2007;73:169-73.

7. Krishna NK, Akshata AA. Surgical removal of complex odontoma-case report. IOSR J Dent Med Sci 2017;16:1-3.

8. Broca P. Traitev Des Tumeurs. Vol. 1. France: P. Asselin; 1866. p. 350.

9. Thoma KH, Goldman HM. Odontogenic tumors: A classification based on observations of the epithelial, 
mesenchymal, and mixed varieties. Am J Patho 1946;22:433-71.

10. Shafer WG, Hine MK, Levy BM. Cysts and tumors of odontogenic origin. In: A Textbook of Oral Pathology. $4^{\text {th }}$ ed. Philadelphia, PA: WB Saunders; 1983. p. 258.

11. Gorlin RJ, Chaudhry AP, Pindborg JJ. Odontogenic tumors. Classification, histopathology, and clinical behavior in man and domesticated animals. Cancer 1961;14:73-101.

12. Ali Azhar D, Kota MZ, El-Nagdy S. An unusual erupted complex composite odontoma: A rare case. Case Rep Dent 2013;2013:106019.

13. Gharote HP, Parate AR. Infected complex odontoma-a case repor. Int J Contemp Med Surg Rad 2016;1:34-6.

14. Lee CH, Park GJ. Complex and compound odontomas are clinico-pathological entities. Basic Appl Pathol 2008;1:30-3.

15. Bhaskar SN. Synopsis of oral pathology. Acad Med 1961;36:845

16. Piattelli A, Perfetti G, Carraro A. Complex odontoma as a periapical and interradicular radiopacity in a primary molar. J Endod 1996;22:561-3.

17. Budnick SD. Compound and complex odontomas. Oral Surg Oral Med Oral Pathol 1976;42:501-6.

18. Kaneko M, Fukuda M, Sano T, Ohnishi T, Hosokawa Y. Microradiographic and microscopic investigation of a rare case of complex odontoma. Oral Surg Oral Med Oral Pathol Oral Radiol Endod 1998;86:131-4.

19. Stafne EC. Value of roentgenograms in diagnosis of tumors of the jaws. Oral Surg Oral Med Oral Pathol 1953;6:82-92.

20. Tejasvi ML, Babu BB. Erupted compound odontomas: A case report. J Dent Res Dent Clin Dent Prospects 2011;5:33-6.

21. Chrcanovic BR, Jaeger F, Freire-Maia B. Two-stage surgical removal of large complex odontoma. Oral Maxillofac Surg 2010;14:247-52.

22. Rumel A, de Freitas A, Birman EG, Tannous LA, Chacon PT, Borkas S. Erupted complex odontoma. Report of a case. Dentomaxillofac Radiol 1980;9:5-9. 